\title{
Modal Vector Fitting: A Tool For Generating Rational Models of High Accuracy With Arbitrary Terminal Conditions
}

\author{
Bjørn Gustavsen, Senior Member, IEEE, and Christoph Heitz
}

\begin{abstract}
This paper introduces a new approach for rational macromodeling of multiport devices that ensures high accuracy with arbitrary terminal conditions. This is achieved by reformulating the vector fitting (VF) technique to focus on eigenpairs rather than matrix elements. By choosing the least squares (LS) weighting equal to the inverse of the eigenvalue magnitude, the modal components are fitted with a relative accuracy criterion. The resulting modal vector fitting (MVF) method is shown to give a major improvement in accuracy for cases with a high ratio between the largest and smallest eigenvalue, although it is computationally more costly than VF. It is also shown how to utilize the impedance characteristics of the adjacent network in the fitting process. The application of MVF is demonstrated for a two-conductor stripline, a coaxial cable, and a transformer measurement. We also show a simplified procedure which achieves similar results as MVF if the admittance matrix can be diagonalized by a constant transformation matrix. The extracted model is finally subjected to passivity enforcement by the modal perturbation method, which makes use of a similar LS formulation as MVF for the constrained optimization problem.
\end{abstract}

Index Terms-Interconnect, macromodel, passivity, pole-residue model, rational model, state-space model, vector fitting.

\section{INTRODUCTION}

W IDEBAND modeling of devices and systems from tabulated data is becoming of major importance for the design and verification of high-speed electronic systems. The modeling is usually based on "fitting" a model to a set of parameters that characterize the model behavior, such as admittance $(\mathbf{Y})$, impedance $(\mathbf{Z})$, or scattering $(\mathbf{S})$ parameters in the frequency domain or the time domain. The fitting process can be based on a ratio of polynomials [1], [2] or orthogonal polynomial functions [3]. Recently, the pole relocating vector fitting technique [4] has become widely applied, and several enhancements have been proposed [5]-[7].

The modeling consists of fitting the model parameters to some given data, minimizing a specific error criterion based on

Manuscript received October 02, 2007; revised October 01, 2008. First published August 19, 2008; current version published November 28, 2008. This work was supported in part by Deutsch, in part by FMC Technologies, in part by Framo, in part by Norsk Hydro, in part by Petrobras, in part by Siemens, in part by Statoil, in part by Total, and in part by Vetco Gray. The work of B. Gustavsen was supported by the Norwegian Research Council (PETROMAKS Programme). The work of C. Heitz was supported by the Swiss Commission of Technology and Innovation CTI. This work was recommended for publication by Associate Editor F. Canavero upon evaluation of the reviewers comments.

B. Gustavsen is with the SINTEF Energy Research, N-7465 Trondheim, Norway (e-mail: bjorn.gustavsen@sintef.no).

C. Heitz is with the Zurich University of Applied Sciences, CH-8401 Winterthur, Switzerland (e-mail: christoph.heitz@ zhaw.ch).

Digital Object Identifier 10.1109/TADVP.2008.927810 a comparison of the model with the given data. In most cases, quadratic error measures are used, leading to a least-squares (LS) fitting. The modeling is complete when the parameters have been fitted to a given accuracy level.

Depending on how the error criterion is constructed, some properties of the physical system are approximated with higher accuracy than others. For instance, when fitting the model to match the elements of a given admittance matrix $(\mathbf{Y})$, the resulting model will yield a good accuracy of the $\mathbf{Y}$-matrix, but not necessarily a good accuracy of the $\mathbf{Z}$-matrix. As we will show below, using this model for calculating currents for given applied voltages will yield accurate results, whereas using it for calculating voltages for given applied currents can generate poor results. Generally speaking, fitting to admittance or impedance parameters yields models that are optimized to a specific terminal condition. Such models may behave unsatisfactory with other terminal conditions. As will be shown in the paper, large error magnifications can take place, depending on the eigenvalue structure.

In this paper, we introduce a more general way of characterizing accuracy by requiring that the model behaves accurately with arbitrary terminal conditions. This is achieved by focusing on the relative accuracy of eigenvalues (modes) rather than matrix elements. This concept is merged with the vector fitting (VF) technique, leading to modal vector fitting (MVF) [8].

The paper is organized as follows. In Section II, we introduce a new type of model error characterization which focuses on the relative accuracy of modal contributions. This concept is merged with the VF methodology in Section IV, leading to MVF. In Section V, it is shown how to take into account the external network since it may render the small eigenvalues of little importance. In Section VI, we introduce a simplified modeling approach which can achieve similar accuracy properties as MVF by usage of a constant transformation matrix. In Section VII, we demonstrate the application of MVF to a stripline transmission line, demonstrating the ability of retaining the accuracy of small eigenvalues. We also show how to retain this accuracy in the subsequent passivity enforcement step by usage of fast modal perturbation (FMP). The shortcomings of assuming a constant transformation matrix are demonstrated in Section VIII for the modeling of a coaxial cable, and the limitations of direct high-order fitting are shown in Section IX.

\section{ACCURACY CONSIDERATIONS}

As an example we consider a multiport system which is described by its admittance parameters. The admittance matrix $\mathbf{Y}$ 
defines the current response $\mathbf{i}$ when applying voltages $\mathbf{v}$ to the ports

$$
\mathbf{i}(s)=\mathbf{Y}(s) \mathbf{v}(s) \text {. }
$$

If current sources are applied to the terminals, the voltage response at any frequency is

$$
\mathbf{v}=\mathbf{Z i}=\mathbf{Y}^{-1} \mathbf{i}=\left(\mathbf{T}_{Y} \mathbf{\Lambda}_{Y} T_{Y}^{-1}\right)^{-1} \mathbf{i}=\mathbf{T}_{Y} \mathbf{\Lambda}_{Y}^{-1} \mathbf{T}_{Y}^{-1} \mathbf{i}
$$

where $\boldsymbol{\Lambda}_{Y}$ is a diagonal matrix holding the eigenvalues of $\mathbf{Y}$, and $\mathbf{T}_{Y}$ is a matrix consisting of the eigenvectors of $\mathbf{Y}$.

Clearly, the small eigenvalues of $\mathbf{Y}$ become the large eigenvalues in $\mathbf{Z}$. If $\mathbf{Y}$ contains both large and small eigenvalues, rational fitting of the elements of $\mathbf{Y}$ is likely to result in a poor representation of the small eigenvalues. Thus, fitting the elements of $\mathbf{Y}$ results in a model that is best suited for reproducing terminal currents if the voltages are given. However, the model is not well suited for reproducing voltages with given currents. Vice versa, fitting the elements of $\mathbf{Z}$ will result in a model that is unable to reproduce correct results if voltages are given. Furthermore, hybrid terminal conditions, imposing currents on some terminals and voltages on other terminals, could result in poor behavior for both of these fitting schemes.

In order to generate an "all-purpose model," we therefore propose to identify the model in such a way that the error associated with each eigenvalue is related to the eigenvalue magnitude by a relative criterion

$$
\left|\frac{\lambda_{i}^{\text {model }}(s)-\lambda_{i}^{\text {data }}(s)}{\lambda_{i}^{\text {data }}(s)}\right|<\epsilon, i=1 \ldots n \text {. }
$$

Usage of this criterion will ensure that the model behaves accurately (with errors in same order of magnitude) with both voltage application and with current application, and with any other (hybrid) terminal condition.

The general problem considered is to identify a pole-residue model $\mathbf{Y}_{\text {rat }}$ (s) (4) in such a way that the accuracy of the modes of $\mathbf{Y}$ is preserved in the relative sense by criterion (3)

$$
\mathbf{Y}(s) \cong \mathbf{Y}_{\text {rat }}(s)=\sum_{m} \frac{\mathbf{R}_{m}}{s-a_{m}}+\mathbf{D}+s \mathbf{E}
$$

The $\left\{a_{m}\right\}$ are the poles of the system and the matrices $\mathbf{R}_{m}$ the corresponding residues. Terms $\mathbf{D}$ and $\mathbf{E}$ are possibly zero.

We assume that $\mathbf{Y}(s)$ is known. The fitting process consists of determining the appropriate model parameters $\left\{a_{m}\right\}, \mathbf{R}_{m}, \mathbf{D}$, and $\mathbf{E}$ for minimizing a suitable error criterion such that (3) is met.

\section{VECTOR FITTING}

Rational fitting of a vectorial frequency response $\mathbf{f}(s)$ by VF [4] amounts to solving the linear problem (5) with a set of predefined poles, $\left\{a_{m}\right\}$

$$
\sigma(s) \mathbf{f}(s) \cong \sum_{m=1}^{N} \frac{\mathbf{r}_{m}}{s-a_{m}}+\mathbf{d}+s \mathbf{e}
$$

$$
\sigma(s)=\sum_{m=1}^{N} \frac{\tilde{r}_{m}}{s-a_{m}}+1 .
$$

Here, $\sigma(s)$ is a scalar function, $\mathbf{f}(s)$ is a column vector of all elements to be fitted, $\left\{a_{m}\right\}$ is the set of common poles, $\mathbf{r}_{m}, \mathbf{d}$ and $\mathbf{e}$ are column vectors of unknown coefficients, and $\left\{\tilde{r}_{m}\right\}$ are additional coefficients specifying the function $\sigma(s)$ (see [4] for details). The columns of the system matrix are scaled to unit length before solving, in order to improve the conditioning.

After solving (5), an improved pole set for $\mathbf{f}(s)$ is calculated as the zeros of $\sigma$, which are computed as the eigenvalues in (6). $\mathbf{A}$ is a diagonal matrix holding the poles $\left\{a_{m}\right\}, \mathbf{b}$ is a column of ones, and $\mathbf{c}$ holds the $\left\{\tilde{r}_{m}\right\}$

$$
\left\{a_{m}\right\}=\operatorname{eig}\left(\mathbf{A}-\mathbf{b c}^{T}\right) .
$$

The new (relocated) poles obtained by (6) are reused in (5) in an iterative procedure. This pole relocation procedure usually converges in a few iterations. In the final step, the residues of the final model are calculated by solving (5a) with $\sigma(s)=1$.

The vector fitting method optimizes the model parameters by minimizing the quadratic error between the given values of the elements of $\mathbf{f}(s)$ and the corresponding model output. For each f $(s)$-element and frequency sample, a specific weight can be introduced for the LS problem [4].

When applying the VF method to fit the elements of a given $\mathbf{Y}$, the elements of the vector $\mathbf{f}(s)$ correspond to the matrix elements $y_{\mathrm{ij}}$ of $\mathbf{Y}$. Since the admittance matrix is symmetric, the length of the vector $\mathbf{f}(\mathrm{s})$ is $n(n+1) / 2$ rather than $n^{2}$.

\section{ModAl Vector FitTing}

We consider a multiport device that is characterized by its admittance matrix $\mathbf{Y}$. This matrix is diagonalized by a (frequency dependent) transformation matrix $\mathbf{T}$ and is to be approximated by a rational model with behavior $\mathbf{Y}_{\text {rat }}$

$$
\mathbf{Y}=\mathbf{T} \Lambda \mathbf{T}^{-1} \cong \mathbf{Y}_{\text {rat }}
$$

Postmultiplying (7) with $\mathbf{T}$ gives for each eigenpair $\left(\lambda_{i}, \mathbf{t}_{i}\right)$

$$
\mathbf{Y}_{\mathrm{rat}} \mathbf{t}_{i} \cong \lambda_{i} \mathbf{t}_{i}, i=1, \ldots, n .
$$

The relative accuracy of the eigenpair is retained in the LS problem by scaling each equation with the inverse of the corresponding eigenvalue magnitude (9)

$$
\frac{1}{\left|\lambda_{i}\right|}\left(\mathbf{Y}_{\mathrm{rat}} \mathbf{t}_{i}-\lambda_{i} \mathbf{t}_{i}\right) \cong 0, i=1, \ldots, n .
$$

Combining (9) with VF leads to MVF. For the pole identification step we get

$$
\begin{array}{r}
\frac{\lambda_{i}}{\left|\lambda_{i}\right|} \sigma(s) \mathbf{t}_{i} \cong \frac{1}{\left|\lambda_{i}\right|}\left(\left(\sum_{m=1}^{N} \frac{\mathbf{R}_{m}}{s-a_{m}}+\mathbf{D}+s \mathbf{E}\right) \mathbf{t}_{i}\right), \\
i=1, \ldots, n .
\end{array}
$$

Equation (10) is built for all modes $i=1, \ldots, n$ and stacked into a common equation. The new (relocated) poles are obtained 


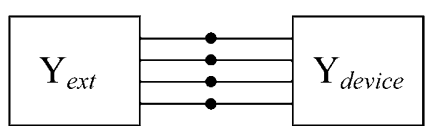

Fig. 1. External network.

from $\sigma(s)$ in (10) by (6), as in the original VF. Finally, the residues are calculated by solving (10) with $\sigma(s)=1$.

In order to improve the convergence properties, the relaxation [6] of the nontriviality constraint is introduced. This frees up the asymptotic term of $\sigma(s)(11)$ while an additional row is added to the system matrix which enforces that the sum of the real part of $\sigma(s)$ over the samples is constant. This row is given an appropriate weighting in the LS problem [6]

$$
\sigma(s)=\sum_{m=1}^{N} \frac{\tilde{r}_{m}}{s-a_{m}}+\tilde{r}_{0} .
$$

The new poles are now calculated by solving the eigenvalue problem (12), where $\mathbf{A}, \mathbf{b}$ and $\mathbf{c}$ are the same as in (6)

$$
\left\{a_{m}\right\}=\operatorname{eig}\left(\mathbf{A}-\mathbf{b} \tilde{r}_{0}^{-1} \mathbf{c}^{T}\right) .
$$

\section{Utilizing External Circuit Properties}

In some situations, the considered device is to be connected to an external network with know impedance characteristics, see Fig. 1. If the admittance seen from the terminals of the device is $\mathbf{Y}_{\text {ext }}$, the total admittance is

$$
\mathbf{Y}_{\text {tot }}=\mathbf{Y}_{\text {device }}+\mathbf{Y}_{\text {ext }} \text {. }
$$

The terminal behavior is now governed by $\mathbf{Y}_{\text {tot }}$ rather than $\mathbf{Y}_{\text {device}}$. This can be utilized in the MVF fitting process by calculating eigenpairs from $\mathbf{Y}_{\text {tot }}$. Equation (10) now becomes

$$
\frac{\lambda_{i}}{\left|\lambda_{i}\right|} \sigma(s) \mathbf{t}_{i} \cong \frac{1}{\left|\lambda_{i}\right|}\left(\left(\mathbf{Y}_{\mathrm{ext}}+\sum_{m=1}^{N} \frac{\mathbf{R}_{m}}{s-a_{m}}+\mathbf{D}+s \mathbf{E}\right) \mathbf{t}_{i}\right) \text {. }
$$

The final computation of residues (with known poles) is done with $\sigma(s)$ in (14) equal to unity.

\section{Modeling VIA CONSTANT TRANSFormation Matrix}

In many applications, the system can be modeled with sufficient accuracy via a constant, real transformation matrix. This offers many advantages since the fitting process involves only the eigenvalues of the admittance matrix.

For instance, a two-conductor interconnect stripline in a symmetrical arrangement leads to balanced matrices for the perunit-length series impedance $\mathbf{Z}(s)$ and shunt admittance $\mathbf{Y}(s)$, i.e., of the form

$$
\mathbf{K}=\left[\begin{array}{ll}
a & b \\
b & a
\end{array}\right] .
$$

TABLE I

ALGORITHM

\begin{tabular}{|l|l|}
\hline Step 1 & $\begin{array}{l}\text { Diagonalize } \mathbf{Y}_{n} \text { using a constant, real } \\
\text { transformation matrix }\end{array}$ \\
\hline Step 2 & Stack the eigenvalues into a single vector \\
\hline Step 3 & $\begin{array}{l}\text { Fit the eigenvalues using a common pole set by } \\
\text { VF, with inverse magnitude weighting }\end{array}$ \\
\hline Step 4 & $\begin{array}{l}\text { Expand the diagonalized model into a pole- } \\
\text { residue model (4) by (19) }\end{array}$ \\
\hline
\end{tabular}

The nodal admittance stamp is of the form

$$
\mathbf{Y}_{n}=\left[\begin{array}{ll}
\mathbf{A} & \mathbf{B} \\
\mathbf{B} & \mathbf{A}
\end{array}\right]
$$

where $\mathbf{A}$ and $\mathbf{B}$ are also balanced matrices. It can be shown that $\mathbf{Y}_{n}$ in (16) is diagonalized by the constant transformation matrix

$$
\mathbf{T}=\left[\begin{array}{cccc}
1 & 1 & 1 & 1 \\
1 & -1 & 1 & -1 \\
1 & 1 & -1 & -1 \\
1 & -1 & -1 & 1
\end{array}\right] / 2
$$

The port admittance matrix $\mathbf{Y}_{n}$ can now be diagonalized using $\mathbf{T}$ in (17)

$$
\Lambda=\mathbf{T}^{-1} \mathbf{Y}_{n} \mathbf{T}
$$

where $\boldsymbol{\Lambda}$ is a diagonal matrix containing the (frequency dependent) eigenvalues of $\mathbf{Y}_{n}$.

Since the diagonalization is done via a constant transformation matrix, each eigenvalue $\lambda_{i}$ represents a linear combination of the matrix elements of $\mathbf{Y}_{n}$. Hence, they contain exactly the same poles as $\mathbf{Y}_{n}$ and are thus suitable for rational fitting. We can therefore stack the modes into a single vector which is subjected to rational fitting by VF. By using inverse magnitude weighting of the LS problem, we achieve that the eigenvalues are fitted to a relative accuracy, thus achieving the same advantages as MVF.

The obtained model can, if desired, be expanded into a poleresidue model (4). Since the eigenvalues have been fitted by a common pole set, we get for each pole the residue matrix

$$
\mathbf{R}_{m}=\mathbf{T} \operatorname{diag}\left\{r_{1, m} \ldots r_{n, m}\right\} \mathbf{T}^{-1}
$$

where $n=4$ for the two-conductor case. The same approach (19) is used for obtaining the constant term $\mathbf{D}$ in (4).

The approach is also applicable in situations with a mild frequency dependency in $\mathbf{T}$. Here, $\mathbf{T}$ is calculated at a fixed frequency point. The eigenvectors are rotated so as to minimize their imaginary parts in the least squares sense [9], and the imaginary part is discarded. The obtained $\mathbf{T}$ is used for calculating an approximate diagonalization of $\mathbf{Y}_{n}$, by (18), and the off-diagonal elements are ignored. (By discarding the imaginary part of $\mathbf{T}$ one achieves that the obtained (approximate) eigenvalues can be accurately fitted with stable poles only). More details on the approach are given in [10].

The procedure is summarized in Table I. 


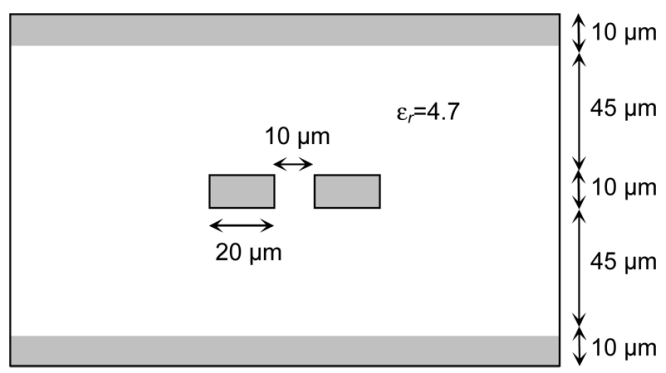

Fig. 2. Stripline interconnect (two conductor).

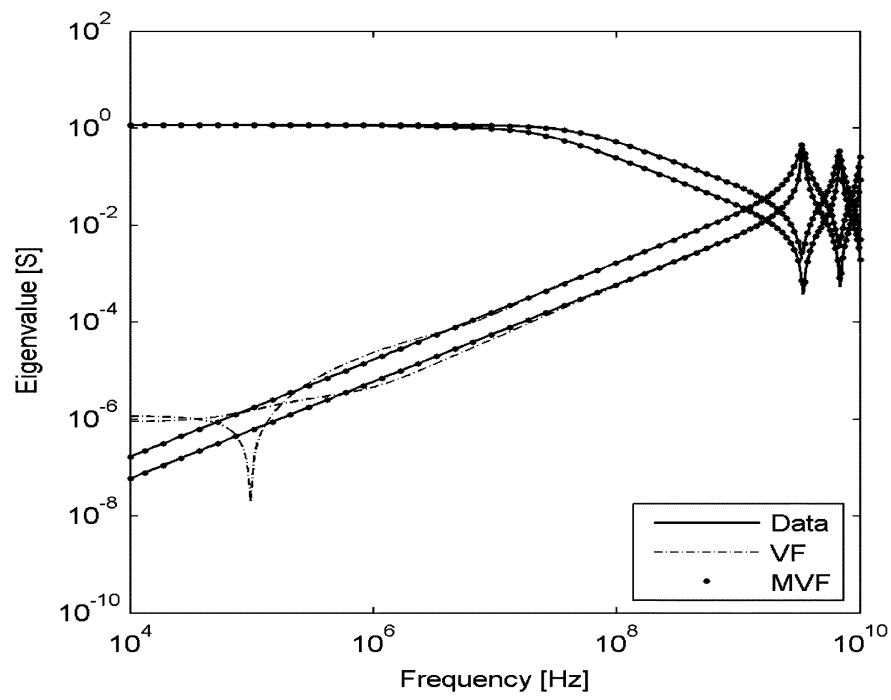

Fig. 3. Eigenvalues of $\mathbf{Y}$.

\section{EXAMPLE: INTERCONNECT MODELING}

\section{A. Modeling via Modal Vector Fitting}

A rational model is to be calculated for a $20-\mathrm{mm}$ stripline with a lossless dielectric, see Fig. 2. The conductivity of the stripline conductors and ground planes is $\sigma=58 E 6 \mathrm{~S} / \mathrm{m}$.

The per-unit-length series impedance was calculated with inclusion of eddy current effects in conductors and ground planes. From the series impedance and shunt admittance, the $4 \times 4$ nodal admittance matrix $\mathbf{Y}(s)$ was established with respect to the line ends. A pole-residue model for $\mathbf{Y}$ was identified in the frequency range $10 \mathrm{kHz}-10 \mathrm{GHz}$ using either VF or MVF (both with relaxation of the nontriviality constraint). In both cases, the fitting process used seven iterations with 18 poles and a nonzero D. With VF, the fitting was done without any user-defined weighting of sample points. This implies that VF attempts at minimizing the global fitting error.

Fig. 3 shows the magnitude of the (complex) eigenvalues of Y. It is observed that when the eigenvalue spread is large (low frequencies), the small eigenvalues become inaccurately represented in the model extracted by VF. With MVF, all eigenvalues are accurately represented at all frequencies, due to the relative criterion (3). This is further shown in Fig. 4 which plots the error deviations by VF and MVF. It is observed with MVF, the deviation curves tend to be parallel to the respective eigenvalues.

Fig. 5 (solid traces) shows the elements of $\mathbf{Y}$ (data) as well as the deviation by the rational models. It is observed that MVF

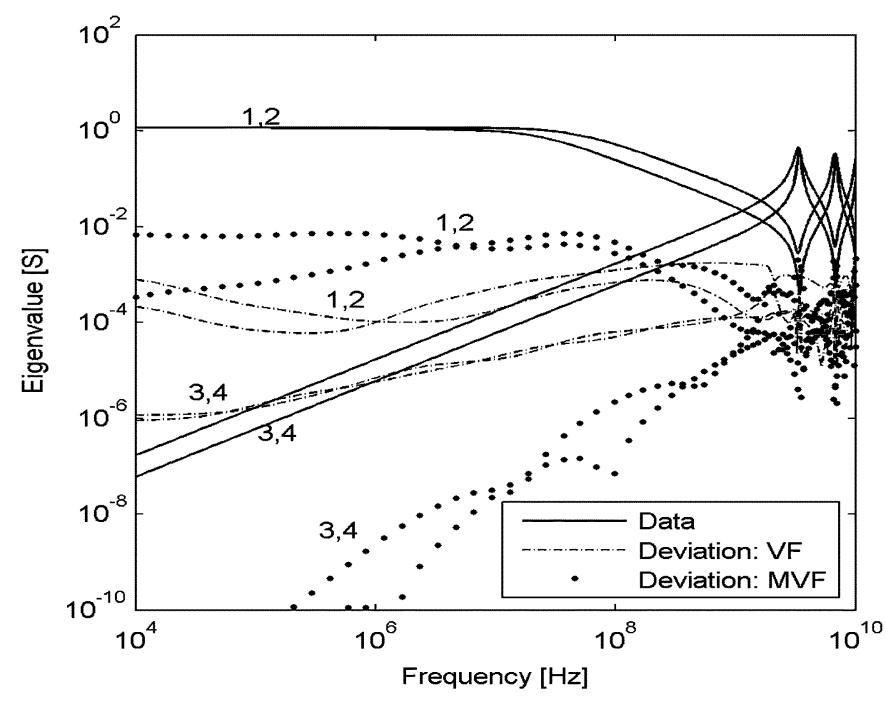

Fig. 4. Eigenvalues of $\mathbf{Y}$. Deviation curves.

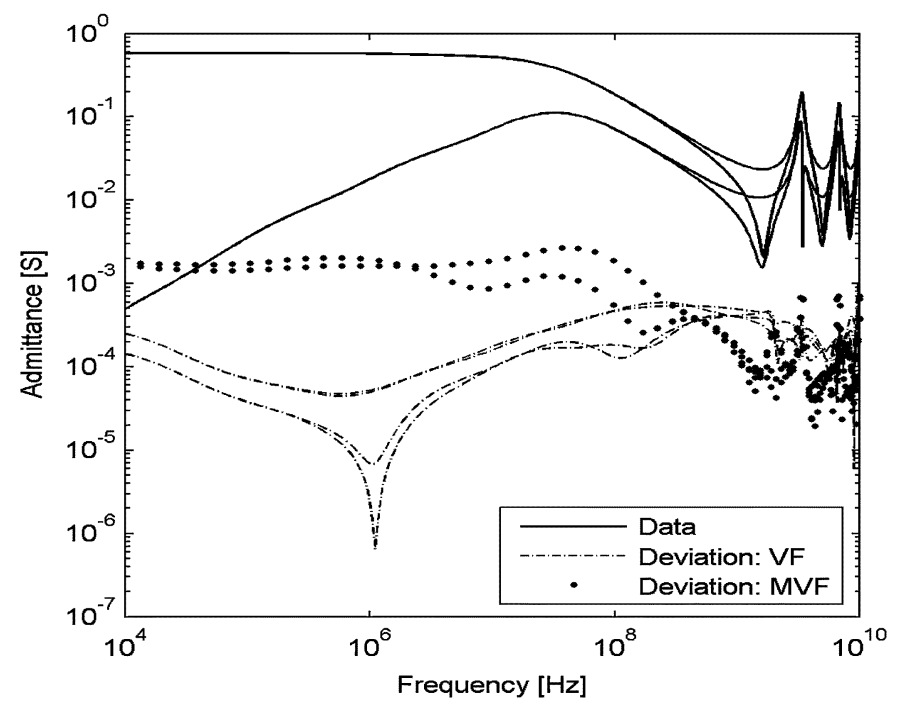

Fig. 5. Elements of $\mathbf{Y}$.

give a higher deviation at low frequencies, which is a consequence of the additional constraint posed by the representation of the small eigenmodes.

Fig. 6 shows the result for $\mathbf{Z}=\mathbf{Y}^{-1}$, which corresponds to the voltage response when applying currents to the line ends. It is seen that the result by MVF remains accurate while that by VF is poor. The latter result is caused by the inability of VF to accurately represent the small eigenvalues, as was shown in Figs. 3 and 4. Since the small eigenvalues of $\mathbf{Y}$ become the large eigenvalues of $\mathbf{Z}$ (2), a catastrophic error magnification takes place. With MVF, the relative accuracy of eigenvalues is preserved and so an accurate result is ensured also for $\mathbf{Z}$.

Fig. 7 compares the fitting error for $\mathbf{Y}$ and $\mathbf{Y}^{-1}$ as function of the number of pole-residue terms, when fitting $\mathbf{Y}$ using either VF or MVF. For each order, the plot shows the maximum of the relative error of the matrix norm, over the given frequency samples (20), (21). When fitting by VF, a (common) weighting equal to $1 /\|\mathbf{Y}(s)\|_{2}$ was applied to all matrix elements, in order to match the error criterion in (20). The result in Fig. 7 shows 


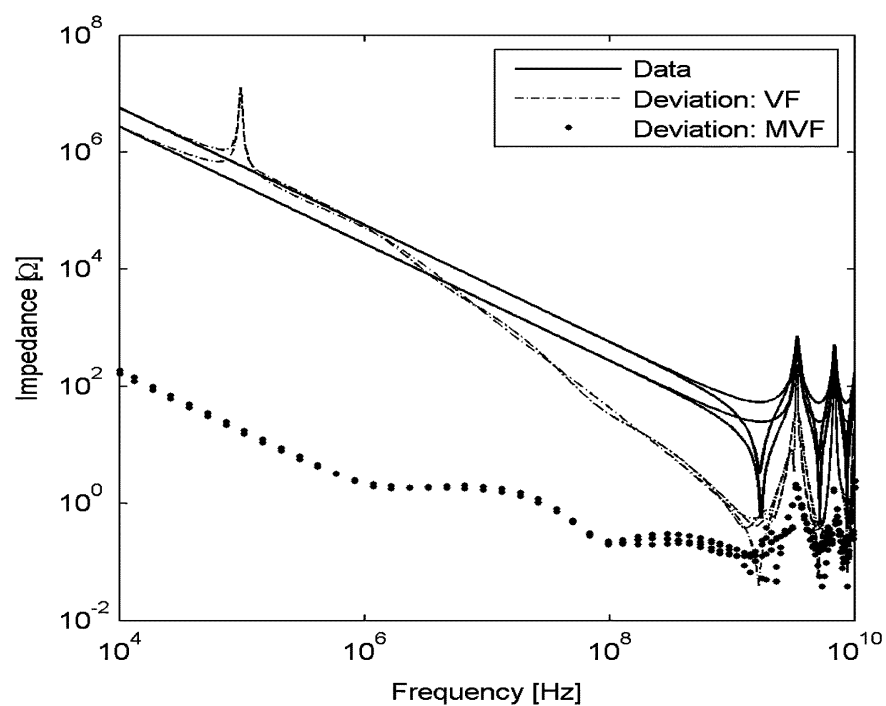

Fig. 6. Elements of $\mathbf{Z}=\mathbf{Y}^{-1}$.

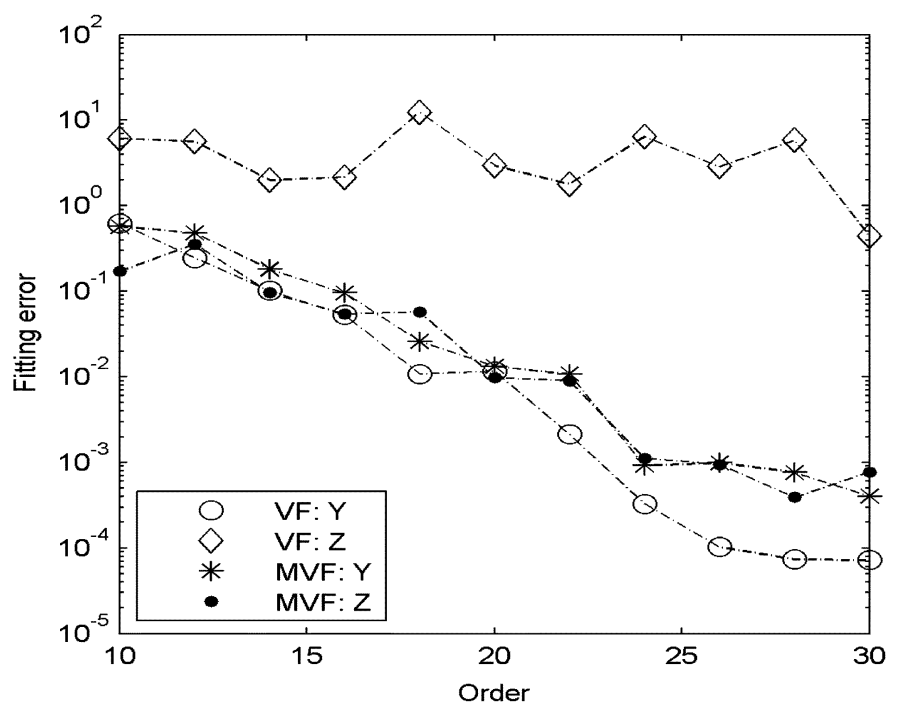

Fig. 7. Fitting error versus model order.

that MVF tends to give an equally accurate representation of $\mathbf{Y}$ and $\mathbf{Z}$, whereas VF only gives a good representation of $\mathbf{Y}$. As explained before, the reason is the inability of VF to represent the (very small) low-frequency eigenvalues of $\mathbf{Y}$, which leads to a catastrophic error magnification in the representation of $\mathbf{Z}=$ $\mathbf{Y}^{-1}$

$$
\begin{aligned}
\epsilon_{Y} & =\max \left(\frac{\left\|\mathbf{Y}_{\mathrm{fit}}(s)-\mathbf{Y}(s)\right\|_{2}}{\|\mathbf{Y}(s)\|_{2}}\right) \\
\epsilon_{Z} & =\max \left(\frac{\left\|\mathbf{Y}_{\mathrm{fit}}^{-1}(s)-\mathbf{Z}(s)\right\|_{2}}{\|\mathbf{Z}(s)\|_{2}}\right) .
\end{aligned}
$$

\section{B. Computational Efficiency}

In the case of multiport systems, the computational bottleneck is the solving of the linear equation $\mathbf{A x}=\mathbf{b}$ associated with the pole identification step, i.e., (5) with VF and (10) with MVF. Fig. 8 shows the sparsity pattern of the system matrix for the pole identification step. We are fitting a symmetric $\mathbf{Y}$ with
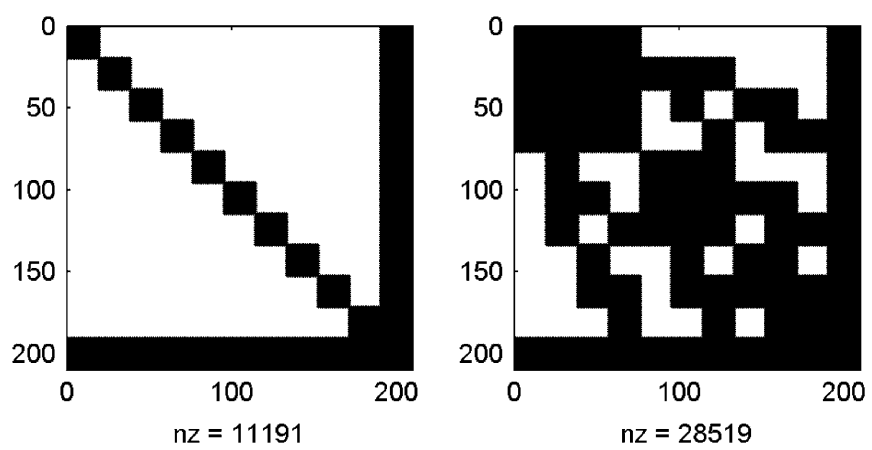

Fig. 8. Sparsity pattern for NEs: VF (left) and MVF (right).

TABLE II

TIME CONSUMPTION [S]

\begin{tabular}{|l|c|c|}
\hline & Normal Equations & QR \\
\hline VF & 0.004 & 2.7 \\
\hline MVF & 0.015 & 5.3 \\
\hline
\end{tabular}

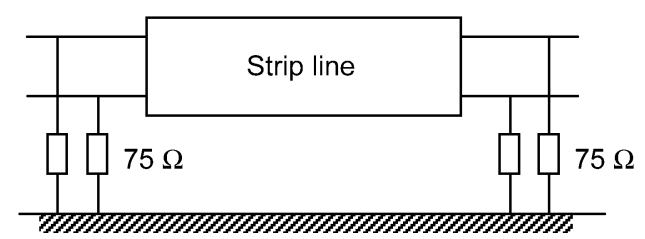

Fig. 9. Connecting stripline to external network.

$n=4$ ports, using $N=18$ pole-residue terms and a nonzero D-term.

- When utilizing symmetry, usage of VF leads to the simultaneous fitting of the upper triangle of the $4 \times 4 \mathrm{Y}$. The vector to be fitted has $n(n+1) / 2=10$ elements. For the pole identification step, this gives 10 blocks of size $N+1=19$ on the diagonal (left panel in Fig. 8). In addition comes the contribution from $\sigma$ which adds another 19 unknowns.

- Usage of MVF leads to $n=4$ blocks on the diagonal of size $n(N+1)=76$. In addition, the contribution from $\sigma$ adds another 19 unknowns. After utilizing symmetry, a smaller size matrix results as shown in Fig. 8, right panel.

The computational cost of solving the equation $\mathbf{A x}=\mathbf{b}$ is higher for MVF than for VF, due to the more unfavorable sparsity pattern. Table II lists the computation time in Matlab (1.3-GHz Pentium processor). It is seen that the computation time using normal equations (NEs) is very small. However, with MVF, the result obtained via NE was found to be inaccurate, possibly because of the (extreme) inverse magnitude scaling in (10). In all calculations, QR was therefore used as solver which leads to a significant computation time (right column in Table II).

\section{Result With Inclusion of External Network}

We proceed with the same example but now assume that the line is going to be terminated at both ends with $75 \Omega$ resistors, see Fig. 9. The fitting of $\mathbf{Y}_{\text {device }}$ by MVF is now done using (14). As before, we use 18 poles with both VF and MVF. 


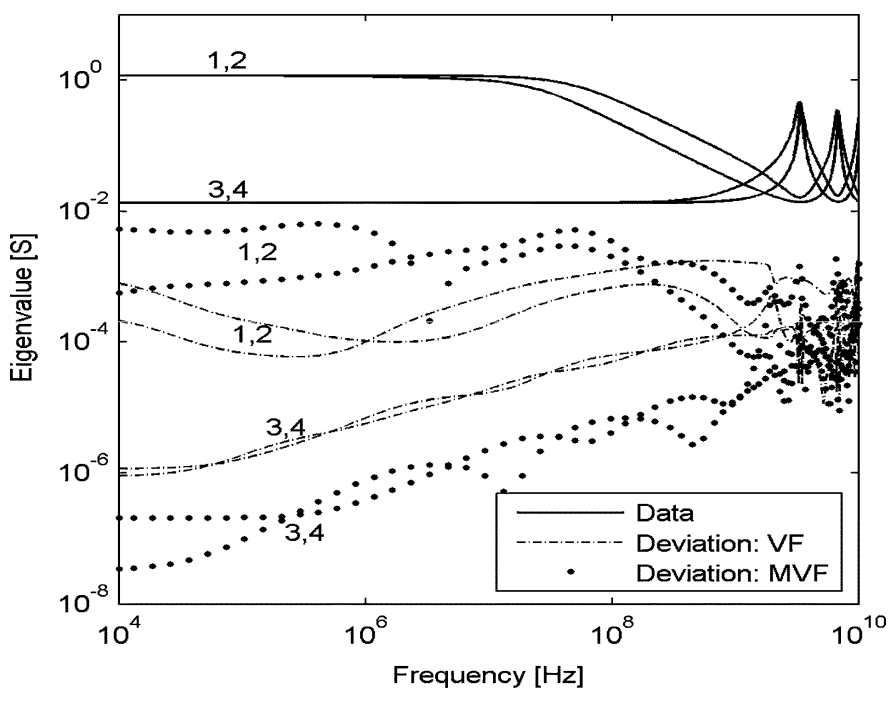

Fig. 10. Eigenvalues of $\mathbf{Y}_{\text {tot }}=\mathbf{Y}_{\text {device }}+\mathbf{Y}_{\text {ext }}$.

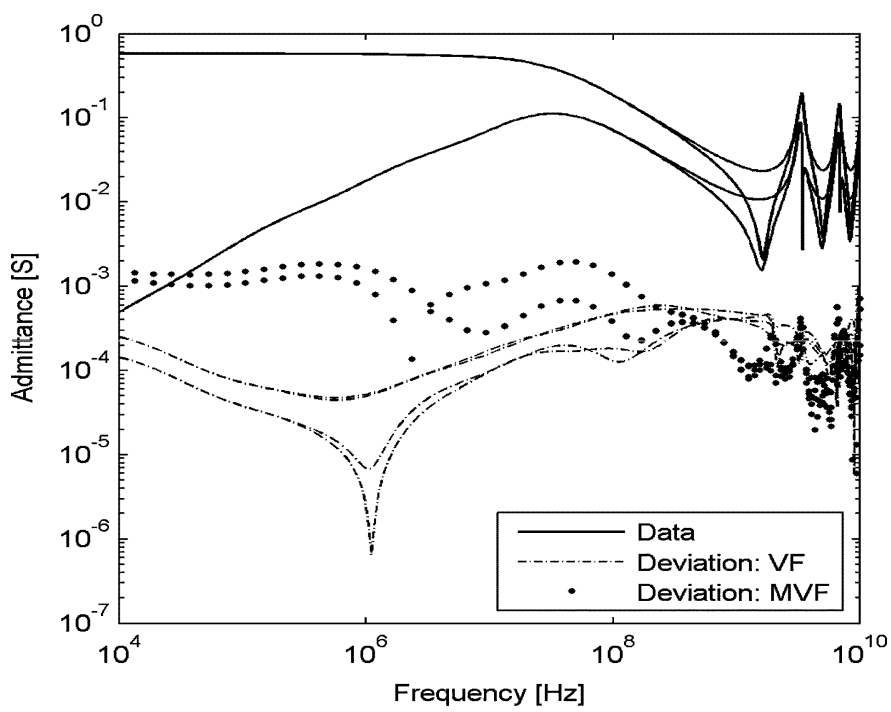

Fig. 11. Elements of $\mathbf{Y}_{\text {device }}$.

The eigenvalues of $\mathbf{Y}_{\text {device }}+\mathbf{Y}_{\text {ext }}$ are shown in Fig. 10 . The eigenvalue spread is now much smaller than in Fig. 3, due to the connection to ground by the $75 \Omega$ resistors. Here, both VF and MVF produce a satisfactory model for the elements of $\mathbf{Y}_{\text {device }}$ (Fig. 11) and $\mathbf{Z}=\left(\mathbf{Y}_{\text {device }}+\mathbf{Y}_{\text {ext }}\right)^{-1}$ (Fig. 12). Still, the model extracted by MVF gives a better representation of $\mathbf{Z}=\left(\mathbf{Y}_{\text {device }}+\mathbf{Y}_{\text {ext }}\right)^{-1}$ than the one obtained by VF, due to the better representation of the small eigenvalues. This comes at the cost of a lower accuracy in the fitting of $\mathbf{Y}_{\text {device }}+\mathbf{Y}_{\text {ext }}$.

\section{Passivity Enforcement}

In general, the extracted model needs to be subjected to passivity enforcement in order to avoid unstable simulations. The most common approach is to perturb the model residues in a postprocessing step, either by quadratic or linear programming [11], [12], or via Hamiltonian matrix theory [13]. Inverse weighting strategies can be applied in order to minimize the relative change to the elements of $\mathbf{Y}$ [14], [15] but this does

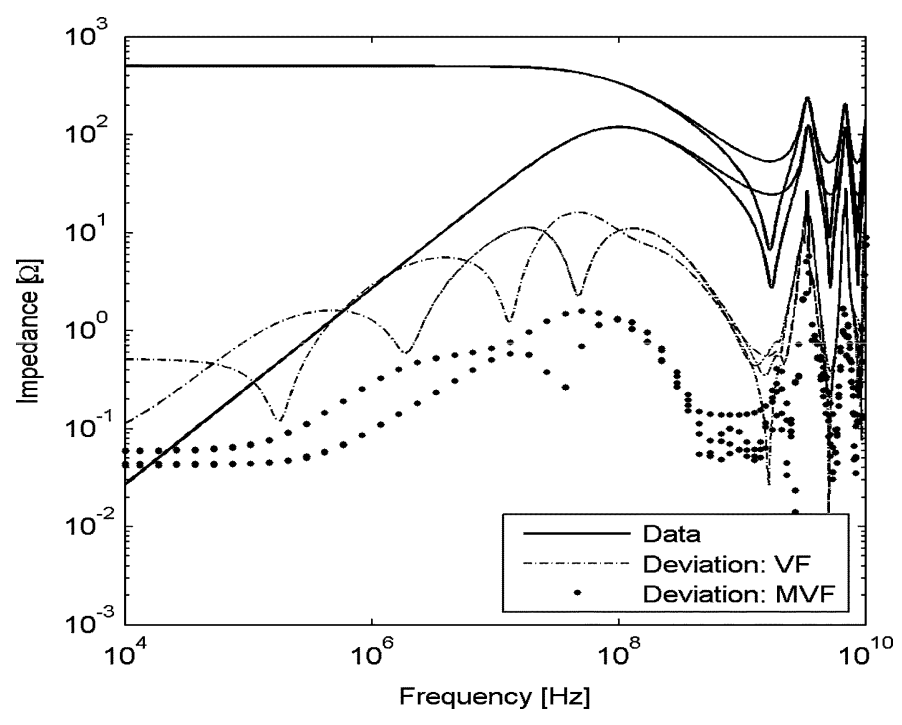

Fig. 12. Elements of $\mathbf{Z}=\left(\mathbf{Y}_{\text {device }}+\mathbf{Y}_{\text {ext }}\right)^{-1}$.

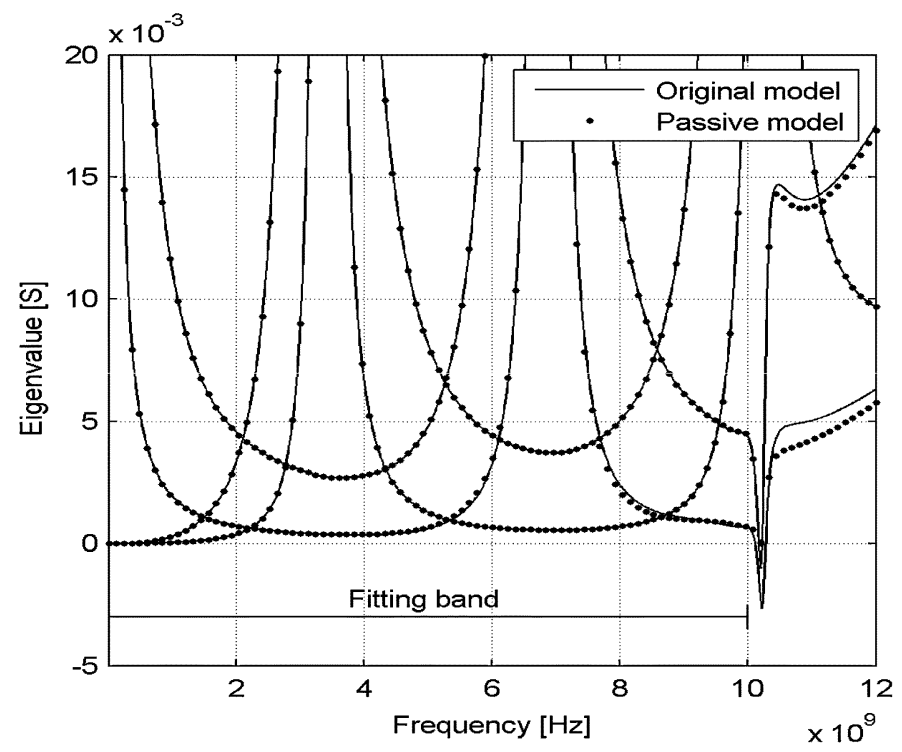

Fig. 13. Eigenvalues of $\mathbf{G}=\operatorname{Re}\{\mathbf{Y}\}$.

not guarantee to prevent that small eigenvalues of $\mathbf{Y}$ become corrupted if a large eigenvalue exists in the same frequency neighborhood (large eigenvalue spread).

In order to retain the relative accuracy of the modes, we therefore use the modal perturbation (MP) technique in [16]. This approach uses the same formulation (9) for the LS-part of the constrained optimization problem (including inverse weighting of eigenvalue magnitude), thereby being a counterpart to MVF. Fast computations are achieved by perturbing only the eigenvalues of the individual residue matrices (FMP). That way, out-of band passivity violations can be corrected without corrupting the small (in-band) eigenvalues of $\mathbf{Y}$.

Fig. 13 shows the eigenvalues of $\mathbf{G}=\operatorname{Re}\{\mathbf{Y}\}$. (G has purely real eigenvalues since it is real and symmetric). It is observed that there are negative eigenvalues at about $10.2 \mathrm{GHz}$, implying passivity violations. The same plot shows that usage of FMP, combined with passivity checking via Hamiltonian 


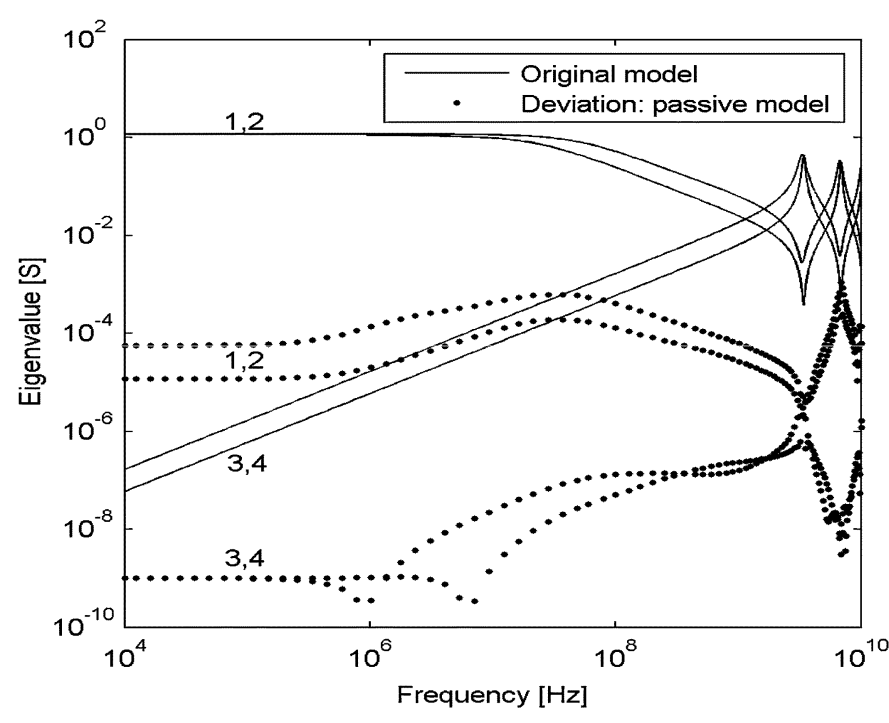

Fig. 14. Impact of passivity enforcement on eigenvalues of $\mathbf{Y}$.

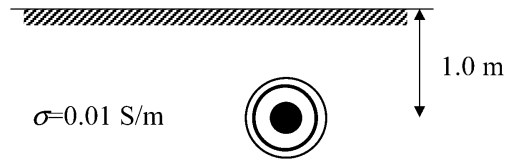

Fig. 15. Coaxial-type underground cable used in power systems.

TABLE III

Cable Data (High-Voltage Cable)

\begin{tabular}{|l|l|}
\hline \multicolumn{1}{|c|}{ Item } & \multicolumn{1}{c|}{ Property } \\
\hline Conductor & $\mathrm{OD}=21.3 \mathrm{~mm}, \sigma=23.9 E 6 \mathrm{~S} / \mathrm{m}$ \\
\hline Insulation & $t=5.5 \mathrm{~mm}, \varepsilon_{\mathrm{r}}=2.3$ \\
\hline Screen & $t=0.35 \mathrm{~mm}, \sigma=58 E 6 \mathrm{~S} / \mathrm{m}$ \\
\hline Jacket & $t=3.5 \mathrm{~mm}, \varepsilon_{\mathrm{r}}=2.3$ \\
\hline
\end{tabular}

matrix eigenvalues and robust iterations, removes the passivity violations without corrupting the in-band behavior.

The impact of the passivity enforcement on the eigenvalues of $\mathbf{Y}$ (magnitudes) is shown in Fig. 14. It is observed that the passivity enforcement does not corrupt the small eigenvalues. The leveling of the small eigenvalues at low frequencies occurs because the FMP-routine attempts at making the eigenvalues of G positive by a small amount (tol $=1 E-9$ ), in order to reduce the required number of iterations. This value $(t o l)$ can be decreased, at the expense of more iterations.

\section{LiMitATions OF APPROACH With CONSTANT TRANSFORMATION MATRIX}

It was verified that for the previous example (stripline interconnect), modeling via a constant transformation matrix (Section VI) gives a similar result as that of MVF. However, there are several situations where the assumption of a constant transformation matrix gives an inaccurate result, due to frequency dependency of the transformation matrix. One example is the modeling of screened cables, see Fig. 15 and Table III.

The terminal admittance matrix $\mathbf{Y}$ was calculated with respect to the core conductor and screen conductor, and subjected

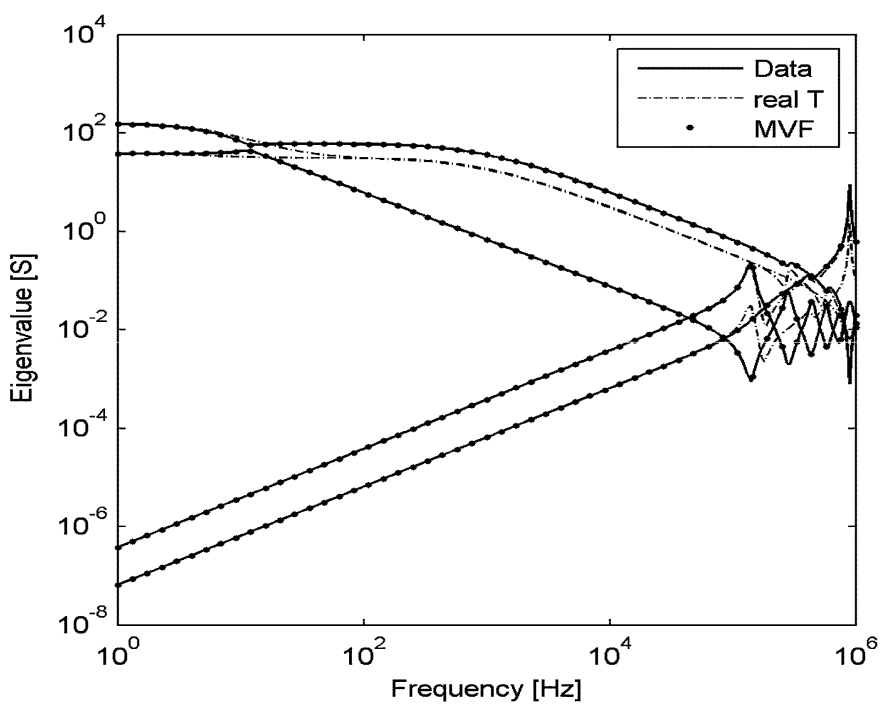

Fig. 16. Eigenvalues of $\mathbf{Y}$.

to rational fitting using MVF. In Fig. 16, it can be seen that the eigenvalues of the MVF model (dots) correspond well with the true eigenvalues (line), indicating that the MVF generates an accurate model of the system. However, when using the constant, real transformation matrix method with $\mathbf{T}$ evaluated at $1 \mathrm{~Hz}$, the model gives good accuracy only at very low frequencies. At higher frequencies, the eigenvalues of the rational model deviate substantially from those of the data. The reason is that the transformation matrix is strongly frequency dependent. This frequency dependency is accounted for when using MVF, but not when applying a constant transformation matrix.

The assumption of a constant transformation matrix will also lead to errors for on-chip interconnects, but they are often smaller than in the cable example. Reference [10] reports the result for a four-conductor interconnect with two alternative conductor configurations.

\section{Limitations of Direct FitTing ApProACH}

An alternative to MVF is to directly fit the elements of $\mathbf{Y}$ using VF with a very high order. The additional degrees of freedom result in a smaller global fitting error and thus a more accurate representation of the small eigenmodes. Unfortunately, the usage of high-order models leads to slower time domain simulations. Another disadvantage is that severe out-of band passivity violations may easily result which can be difficult to correct without corrupting the in-band behavior.

Furthermore, there are situations where the direct fitting approach is not applicable. Reference [17] describes a modeling procedure based on a direct measurement of the elements of a transformer admittance matrix. In order to accurately represent the small eigenvalues that correspond to the so-called zero sequence system, they are measured separately. The zero-sequence modes are then merged into the measured $\mathbf{Y}$. As a result, the obtained $\mathbf{Y}$ is both noisy (due the measurement), and it has a large eigenvalue spread.

Fig. 17 shows a sixtieth-order rational fitting of the $(3 \times 3)$ matrix partition of $\mathbf{Y}$ that corresponds to the delta-winding. It appears that usage of the direct fitting approach and MVF gives 


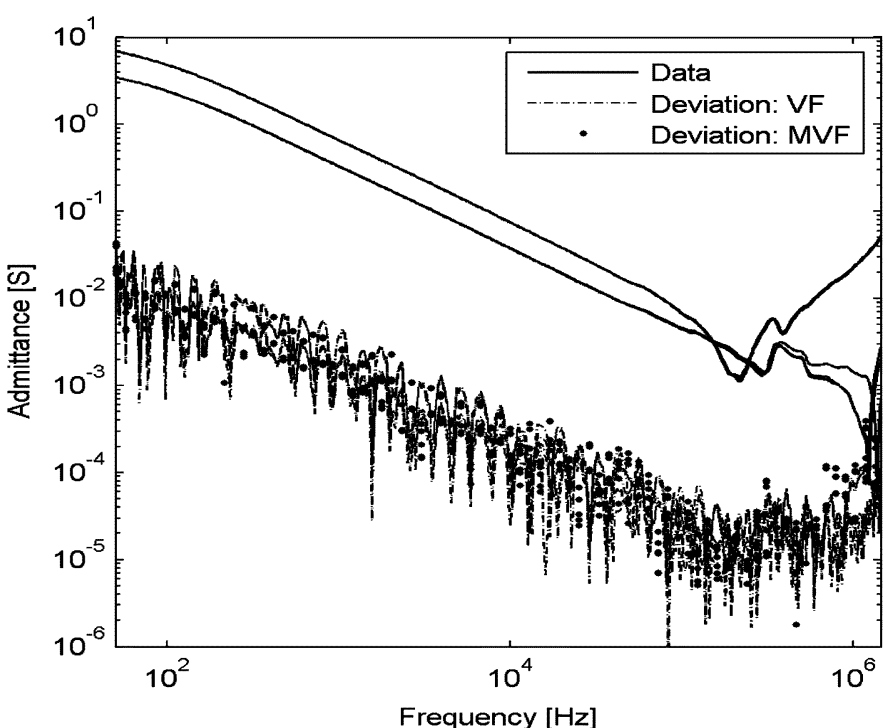

Fig. 17. Fitted admittance matrix.

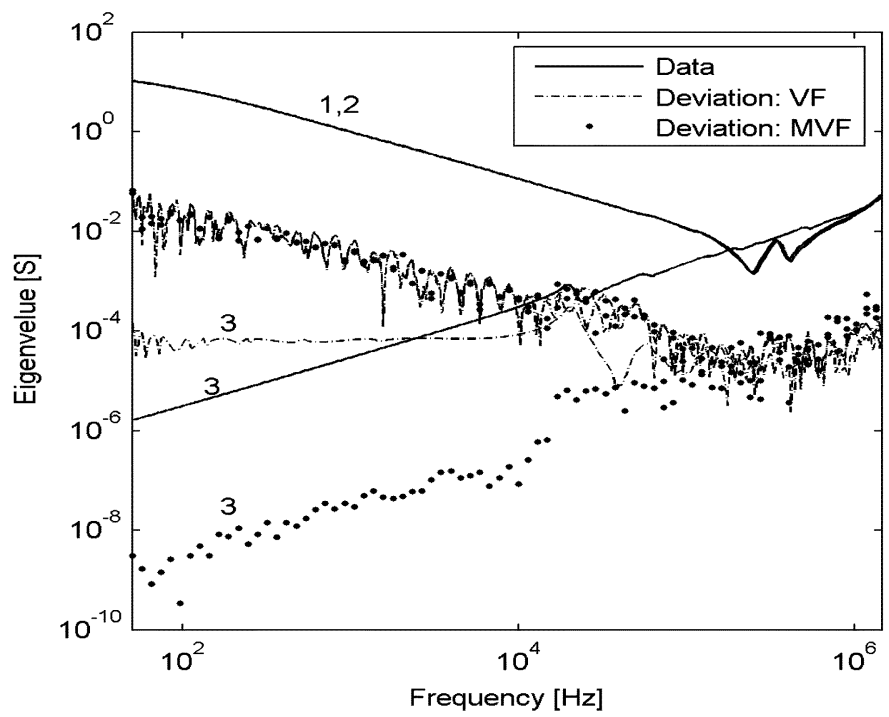

Fig. 18. Eigenvalues of fitted admittance matrix (VF versus MVF).

a similar result in terms of accuracy. However, the plot of eigenvalues in Fig. 18 reveals that MVF is able to accurately extract the small eigenvalue buried in the (noisy) $\mathbf{Y}$, whereas the direct fitting gives an inaccurate result. A further increase of the fitting order does not help since the direct fitting approach cannot distinguish between the noise and the small eigenvalue.

\section{DISCUSSION}

This paper has shown that fitting a rational model to admittance parameters leads to a model which behaves accurately with voltage applications, whereas substantial error magnification can occur with current applications, unless the relative accuracy of the admittance matrix modes is retained. One might think that current applications do not occur in practice, but any situation with open terminals involves current applications. This is because an open terminal implies that zero current is applied to that terminal. As a result, a partition of the admittance matrix is effectively being inverted in the simulation, and error magnifications will occur if that partition has a large eigenvalue spread.

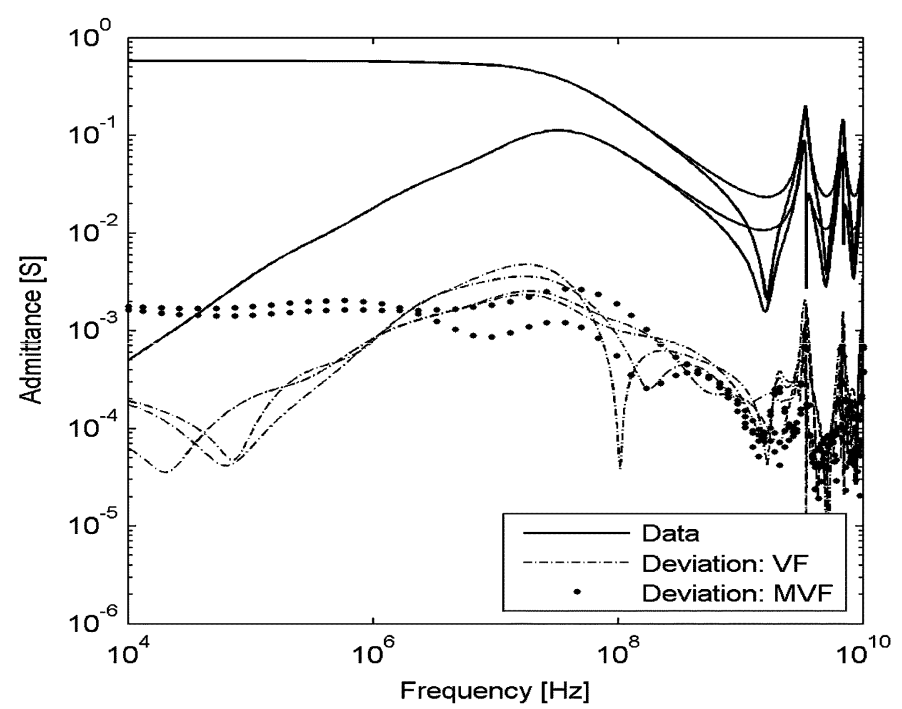

Fig. 19. Elements of $\mathbf{Y}$. VF with relative error control.

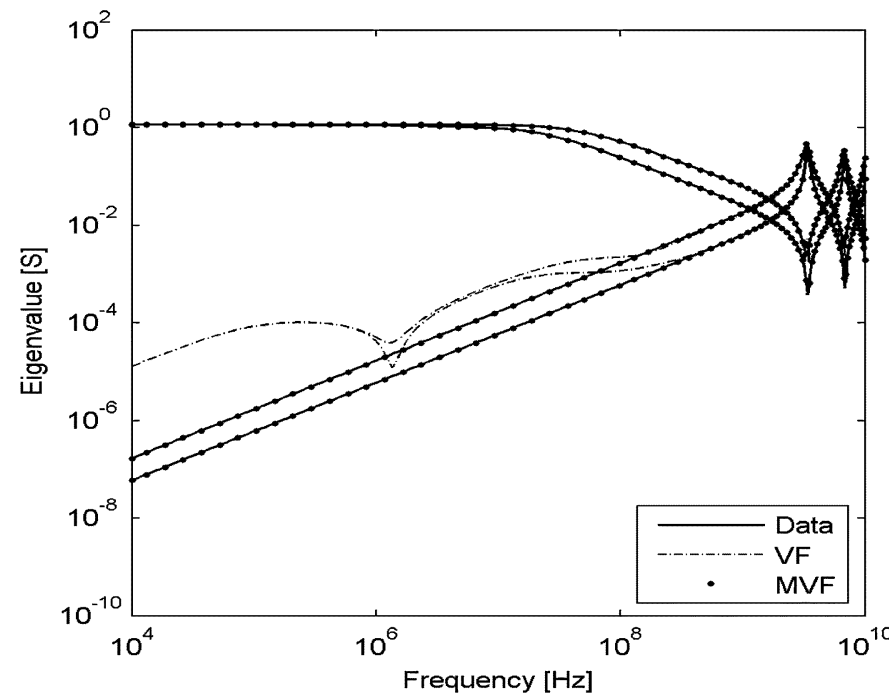

Fig. 20. Eigenvalues of $\mathbf{Y}$. VF with relative error control.

For the stripline example in Section VII, a simulation involving three open terminals will always lead to error magnifications at low frequencies (unless the relative accuracy of eigenvalues is preserved). Usage of MVF ensures that the model behaves accurately in all situations. The current implementation of MVF is best suited for cases with a relatively low number of ports as the computational efficiency is lower than for VF.

The result by VF can be manipulated by introducing weighting (row-scaling) in the least squares problem (5). Fig. 19 shows the effect of applying inverse magnitude weighting to individual elements in the fitting process (eighteenth-order approximation). This gives a rational fitting which attempts at minimizing the relative errors in the elements of $\mathbf{Y}$ (compare Fig. 19 with Fig. 5). Although such weighting can improve the result, it did not prevent the small eigenvalues of $\mathbf{Y}$ from being corrupted, see Fig. 20.

The smallest eigenvalues of an admittance model may become unimportant when the model is embedded in a network. Knowledge about the input admittance seen into the external network can be directly utilized in the MVF process as shown 
in Sections V and VII-C. That way, one avoids that extremely small (but unimportant) eigenvalues are accurately fitted, which would lead to an unnecessary high fitting order.

Sometimes, the device to be modeled has certain symmetry properties that makes it possible to diagonalize $\mathbf{Y}$ via a constant, real transformation matrix (Section VI). Here, the eigenvalues can be fitted independently, and usage of VF with inverse magnitude weighting leads to a model with accurately represented eigenvalues, similarly as the one extracted by MVF. This approach was possible for the two-conductor interconnect in Fig. 2, but failed for a coaxial cable example (Section VIII) as the modal transformation matrix was strongly frequency dependent.

An alternative to MVF is to apply VF directly to the matrix elements of $\mathbf{Y}$, increasing the order until the smallest eigenvalues are accurately represented. Besides increasing the fitting order substantially compared to MVF, the extracted model will often have large out-of-band passivity violations due to over-fitting. Furthermore, situations exist where the direct fitting approach is not applicable, for instance the transformer example in Section IX. Here, the modal contributions of a measured $\mathbf{Y}$ had noise levels that were correlated with the eigenvalue magnitude. This made a direct fitting approach impossible since the small eigenvalue could not be distinguished from the noise.

It is essential that the subsequent passivity enforcement will not corrupt the small eigenvalues, or the advantages of using MVF are lost. Such corruption is avoided by application of fast modal perturbation (FMP) [16]. This approach makes use of inverse magnitude weighting of the modes of $\mathbf{Y}$ in the LS part of the constrained optimization problem, similarly as in MVF. This implies that the eigenvalues of $\mathbf{Y}$ tend to be perturbed in relation to their magnitude. It was shown in Section VII-D that an out-of-band passivity violation was successfully corrected without corrupting the in-band behavior of any eigenvalue.

As a final remark we note that for the fitting of scalar responses (single element), the MVF approach becomes equal to VF with inverse magnitude weighting.

\section{CONCLUSION}

A reformulated, MVF has been developed that ensures high accuracy of the obtained model with arbitrary terminal conditions. This is achieved by explicitly introducing eigenpairs in the modeling, thereby allowing to ensure high relative accuracy for all modal components. Application to examples with a high ratio between the largest and smallest eigenvalue has demonstrated superior accuracy over the traditional approach of fitting matrix elements. The MVF approach also allows to take into account the impedance characteristics of the adjacent network. Compared to the traditional VF approach, MVF is computationally more expensive due to a less favorable sparsity structure for the system equation.

\section{ACKNOWLEDGMENT}

The authors would like to thank Dr. S. Grivet-Talocia and M. Bandinu (Politecnico di Torino, Italy) for providing the perunit-length parameters of the stripline example in Section VII.

\section{REFERENCES}

[1] E. C. Levi, "Complex-curve fitting," IRE Trans. Autom. Control, vol. AC-4, pp. 37-44, 1959.

[2] C. K. Sanathanan and J. Koerner, "Transfer function synthesis as a ratio of two complex polynomials," IEEE Trans. Autom. Control, vol. AC-8, no. 1, pp. 56-58, Jan. 1963.

[3] C. P. Coelho, J. R. Phillips, and L. M. Silveira, "Generating high-accuracy simulation models using problem-tailored orthogonal polynomials basis," IEEE Trans. Circuits Syst. I, vol. 53, no. 12, pp. 2705-2714, Dec. 2006.

[4] B. Gustavsea and A. Semlyen, "Rational approximation of frequency domain responses by vector fitting," IEEE Trans. Power Del., vol. 14, no. 3, pp. 1052-1061, Jul. 1999

[5] S. Grivet-Talocia, "Package macromodeling via time-domain vector fitting," IEEE Microw. Wireless Compon. Lett., vol. 13, no. 11, pp. 472-474, Nov. 2003

[6] B. Gustavsen, "Improving the pole relocating properties of vector fitting," IEEE Trans. Power Del., vol. 21, no. 3, pp. 1587-1592, Jul. 2006.

[7] D. Deschrijver, B. Haegeman, and T. Dhaene, "Orthonormal vector fitting: A robust macromodeling tool for rational approximation of frequency domain responses," IEEE Trans. Adv. Packag., vol. 30, no. 2, pp. 216-225, May 2007.

[8] B. Gustavsen and C. Heitz, "Rational modeling of multi-port systems by modal vector fitting," in Proc. 11th IEEE Workshop Signal Propagation Interconnects, Genova, Italy, May 13-16, 2007, pp. 49-52.

[9] V. Brandwajn, "Modifications of user's instructions for "MARTI SETUP"," EMTP Newsletter, vol. 3, no. 1, pp. 76-80, Aug. 1982.

[10] B. Gustavsen, "Fast delay-less interconnect macromodeling and simulation by assumption of a constant eigenvector matrix," in Proc. 12th IEEE Workshop Signal Propagation Interconnects, May 2008, pp. 1-4.

[11] B. Gustavsen and A. Semlyen, "Enforcing passivity for admittance matrices approximated by rational functions," IEEE Trans. Power Syst., vol. 16, no. 1, pp. 97-104, Feb. 2001.

[12] D. Saraswat, R. Achar, and M. Nakhla, "A fast algorithm and practical considerations for passive macromodeling of measured/simulated data," IEEE Trans. Adv. Packag., vol. 27, no. 1, pp. 57-70, Feb. 2004.

[13] S. Grivet-Talocia, "Passivity enforcement via perturbation of hamiltonian matrices," IEEE Trans. Circuits Syst. I, Fundam. Theor. Appl., vol. 51, no. 9, pp. 1755-1769, Sep. 2004.

[14] B. Gustavsen, "Computer code for passivity enforcement of rational macromodels by residue perturbation," IEEE Trans. Adv. Packag., vol. 30, no. 2, pp. 209-215, May 2007.

[15] S. Grivet-Talocia, "Passivity enforcement with relative error control," IEEE Trans. Microwave Theory Tech., vol. 55, no. 11, pp. 2374-2383, Nov. 2007.

[16] B. Gustavsen, "Fast passivity enforcement of rational macromodels by perturbation of residue matrix eigenvalues," in Proc. 11th IEEE Workshop Signal Propagation Interconnects, Genova, Italy, May 13-16, 2007, pp. 71-74.

[17] B. Gustavsen, "Frequency-dependent modeling of power transformers with ungrounded windings," IEEE Trans. Power Del., vol. 19, no. 3, pp. 1328-1334, Jul. 2004.

Bjørn Gustavsen (M'94-SM'03) was born in Norway, in 1965. He received the M.Sc. degree and the Dr.Ing. degree from the Norwegian Institute of Technology (NTH), Trondheim, Norway, in 1989 and 1993, respectively.

Since 1994 he has been with SINTEF Energy Research. His interests include simulation of electromagnetic transients and modeling of frequency dependent effects. He spent 1996 as a Visiting Researcher at the University of Toronto, Toronto, ON, Canada, and the summer of 1998 at the Manitoba HVDC Research Centre, Winnipeg, MB, Canada. He was a Marie Curie Fellow at the University of Stuttgart, Stuttgart, Germany from August 2001 to August 2002.

Christoph Heitz was born in 1965. He received the Ph.D. degree in theoretical physics from the University of Freiburg, Germany, in 1996.

During 1997-2000, he was a researcher in the field of high-voltage engineering at ABB Corporate Research, Baden-Dättwil, Switzerland. Since 2000, he has been a Professor at the Zurich University of Applied Sciences, Institute for Data Analysis and Process Design, Switzerland. His research interests include signal and data analysis of complex technical systems. For the academic year 2007-2008, he is a Visiting Professor at the School of Engineering, University of California, Santa Cruz. 